\title{
Pancytopenia with Mediastinal Mass: Clinically Challenging at Times
}

\author{
Hasan AAL-Yaseen ${ }^{\mathrm{a}}$ Mahmoud Marashi $^{\mathrm{a}} \quad$ Asma Sultan Al Olama $^{\mathrm{a}}$ \\ Rania Seliem ${ }^{b}$ \\ ${ }^{a}$ Dubai Health Authority, Hematology Department, Dubai Hospital, Dubai, United Arab Emirates; \\ ${ }^{b}$ Dubai Health Authority, Laboratory Department, Rashid Hospital, Dubai, United Arab Emirates
}

\section{Keywords}

Extramedullary hematopoiesis - Thalassemia .

Paramediastinal mass $\cdot$ Pancytopenia $\cdot$ Hydroxyurea

\begin{abstract}
Ineffective erythropoiesis (IE), occurring during chronic bone marrow failure (chronic hemolytic conditions or bone marrow infiltration by neoplastic or fibrotic tissue), results in several compensatory mechanisms, such as erythroid marrow expansion and extramedullary hematopoiesis (EMH). EMH can involve different body organs. We report on a 16-yearold male, previously known to have thalassemia minor, who was admitted with chest tightness and dry cough. He was found to have right-sided pleural effusion and hepatosplenomegaly. The full blood count demonstrated mild pancytopenia. Computed tomography (CT) scan demonstrated the presence of right-sided pleural effusion and a paramediastinal mass. The initial clinical impression was mainly lymphoma. CT-guided needle biopsy of the paramediastinal mass revealed trilineage hematopoiesis with marked erythroid hyperplasia, consistent with EMH. Hemoglobin electrophoresis was consistent with thalassemia intermedia. This illustrates how the presence of EMH can be clinically misleading.

(c) 2019 The Author(s)

Published by S. Karger AG, Basel
\end{abstract}

\section{Introduction}

Extramedullary hematopoiesis (EMH) in thalassemic patients is commonly found in the liver and spleen. However, it might also involve other organs, such as the pleura, lungs, gastrointestinal tract, breast, skin, central nervous system, kidneys, and adrenal glands. The clinical presentation might mimic other space-occupying lesions affecting these tissues and organs. It is important to recognize the possibility of EMH as a differential diagnosis of such clinical presentations, especially in patients with hemoglobinopathies, myeloproliferative neoplasms, or chronic hemolytic anemia. No evidence-based guidelines are available for the treatment of paramediastinal EMH.

\section{Case Presentation}

A 16-year-old male from the United Arab Emirates (UAE), previously thought to have thalassemia minor, presented to our hospital after 2 weeks of fever, chest tightness, and dry cough. He denied shortness of breath, but chest tightness had been progressive over the last 2 weeks before admission. He had attended a private hospital where he was found to have pancytopenia and rightsided pleural effusion. A pleural tap was done, and he was given a blood transfusion in the private hospital. The family requested moving the patient to the hematology unit of a government hospital. The patient did not complain about palpitations or postural

\begin{tabular}{ll}
\hline KARGER & $\begin{array}{l}\text { () } 2019 \text { The Author(s) } \\
\text { Published by S. Karger AG, Basel Oper }\end{array}$ \\
E-Mail karger@karger.com & This article is licensed under the Creative Commons Attribution- \\
www.karger.com/dmj & $\begin{array}{l}\text { NonCommercial-NoDerivatives 4.0 International License (CC BY- } \\
\text { NC-ND) (http://www.karger.com/Services/OpenAccessLicense). } \\
\text { Usage and distribution for commercial purposes as well as any dis- } \\
\text { tribution of modified material requires written permission. }\end{array}$
\end{tabular}

Hasan Mohammed Ali AAL-Yaseen

Dubai Health Authority, Hematology Department, Dubai Hospital 7272, Dubai (United Arab Emirates)

E-Mail alyaseen.hasan@gmail.com 


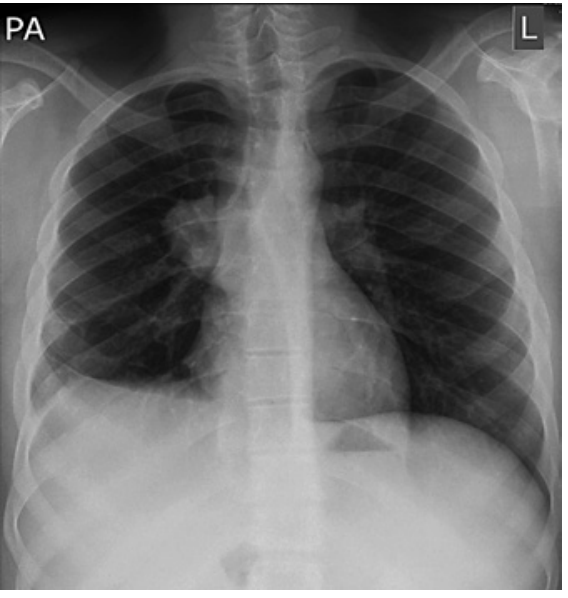

Fig. 1. Plain chest X-ray of the patient showing mediastinal mass and right-sided pleural effusion.

dizziness. There was no history of loss of weight, profuse sweating, skin rash, or joint and bone pain. He did not report episodes of bleeding from any site. There was no history of other systemic symptoms. The patient had received no previous blood transfusion. Otherwise, the patient has no chronic medical disorder, does not consume any drug regularly, and has no known drug allergies. His family has a history of thalassemia; the patient has siblings (1 brother and 2 sisters) with thalassemia, but they have never received blood and are not regularly followed-up by any physician. The patient is a student in the ninth grade. He does not smoke or consume alcohol.

His vital signs at presentation were stable, yet he was slightly febrile at $37.7^{\circ} \mathrm{C}$. He appeared pale with a tinge of jaundice. Lymph nodes were not palpable. His oral cavity was clear. No stigmata of chronic liver disease were found. Chest examination revealed a dull percussion note, and absent breath sounds on the right side posteriorly. Abdominal examination revealed hepatosplenomegaly. The liver span was $16.6 \mathrm{~cm}$ and the spleen span $19.6 \mathrm{~cm}$. There was no ascites. The examination of the cardiovascular and nervous system was normal.

\section{Investigation}

The initial blood tests detected mild pancytopenia with a white blood cell (WBC) count of $3.1 \times 10^{9} / \mathrm{L}$, hemoglobin $(\mathrm{Hb}) 8.5 \mathrm{~g} / \mathrm{dL}$, mean corpuscular volume $71 \mathrm{fL}$, and platelet count $94 \times 10^{9} / \mathrm{L}$. Morphology of the peripheral blood was leucoerythroblastic with polychromasia, suggestive of bone marrow stress or infiltration. The reticulocyte count was 5\%, lactate dehydrogenase was $948 \mathrm{U} / \mathrm{L}$ (ref. range 240-480 U/L), and the direct Coombs test was negative. Total serum bilirubin was $3.2 \mathrm{mg} / \mathrm{dL}$. The other liver function tests were normal. The coagulation profile was indicative of very mild prolongation of prothrombin time at $15 \mathrm{~s}$, with an INR of 1.3. His renal function test was normal. Screening for HIV, HBsAg, and hepatitis $\mathrm{C}$ was negative. A Hb electrophoresis test was performed in the private hospital before transfusion, showing $\mathrm{HbA}$ of $28.6 \%$, $\mathrm{HbF} 58.1 \%$, and $\mathrm{HbA}_{2} 3.3 \%$. This pattern is consistent with thalassemia intermedia. Chest X-ray findings indicated right-sided pleu-

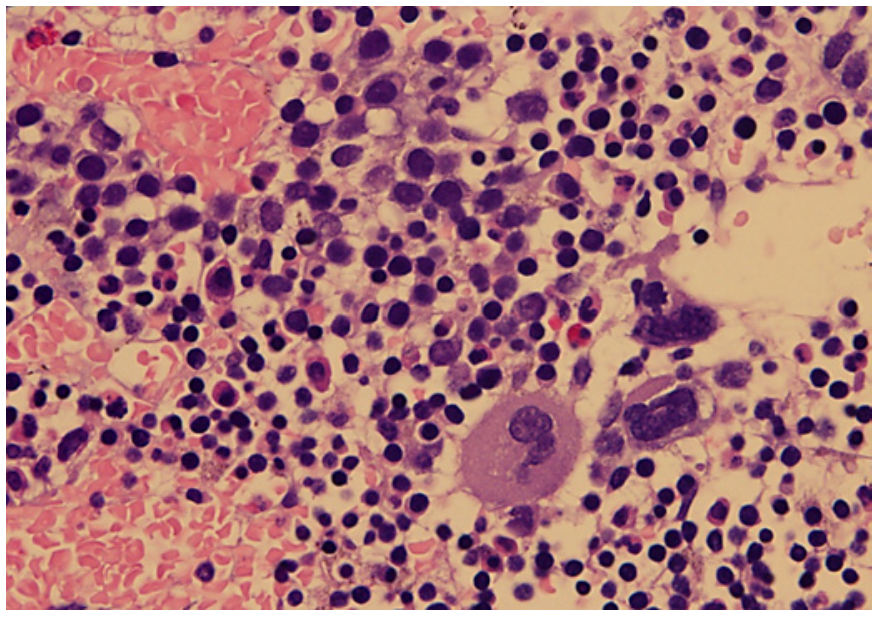

Fig. 2. Mediastinal mass biopsy demonstrating marked erythroid hyperplasia.

ral effusion with underlying consolidation collapse (Fig. 1). His parents requested not to repeat the computed tomography (CT) scan as it had been done in the private hospital showing a mediastinal mass, right-sided pleural effusion, and hepatosplenomegaly. No enlargement of other lymph node groups was detected by CT scan. Meanwhile, the patient was clinically stable apart from feeling increasing chest tightness. He was managed symptomatically. A CT-guided fine needle biopsy of the paramediastinal mass was done. An intercostal drain was inserted at the same time to drain the pleural fluid, for diagnostic and therapeutic purposes. The pleural fluid was hemorrhagic and exudative. Pleural fluid protein was $4.7 \mathrm{~g} / \mathrm{dL}$. The microbiology report of the pleural fluid stated that the sample was unsuitable for cell counting and differential diagnosis because it was totally bloody. No organisms were seen by gram stain. The ordinary and TB cultures of the pleural fluid showed no growth. Cytology smears of the pleural fluid demonstrated few scattered mesothelial cells. The background showed inflammatory cells composed of few neutrophils, lymphocytes, eosinophils, and macrophages admixed with abundant red blood cells (RBC). Flow cytometry of the pleural fluid was run to rule out clonal disease or lymphoproliferative disorder. It was shown that the effusion was reactive, with no evidence of clonality. The patient improved symptomatically after insertion of the intercostal drain, but developed a fever of $38.6^{\circ} \mathrm{C}$. His $\mathrm{Hb}$ level dropped further. Creactive protein was $>120 \mathrm{mg} / \mathrm{L}$, but the blood culture was negative for growing microbes. He was empirically treated with piperacillin/tazobactam and given a blood transfusion. The fever subsided after a few days, and he felt symptomatically better.

The gross appearance of the biopsy from the mediastinal mass was blackish, as reported by the interventional radiologist. Histology of the mediastinal mass demonstrated trilineage hematopoiesis with marked erythroid hyperplasia and mild histiocytes hyperplasia (Fig. 2).

Bone marrow aspiration demonstrated trilineage hematopoiesis and marked erythroid hyperplasia, with several abnormal histiocytes. No histiocytic clusters were seen. There were no granulomas and no hemophagocytosis. Flow cytometry of the bone mar- 


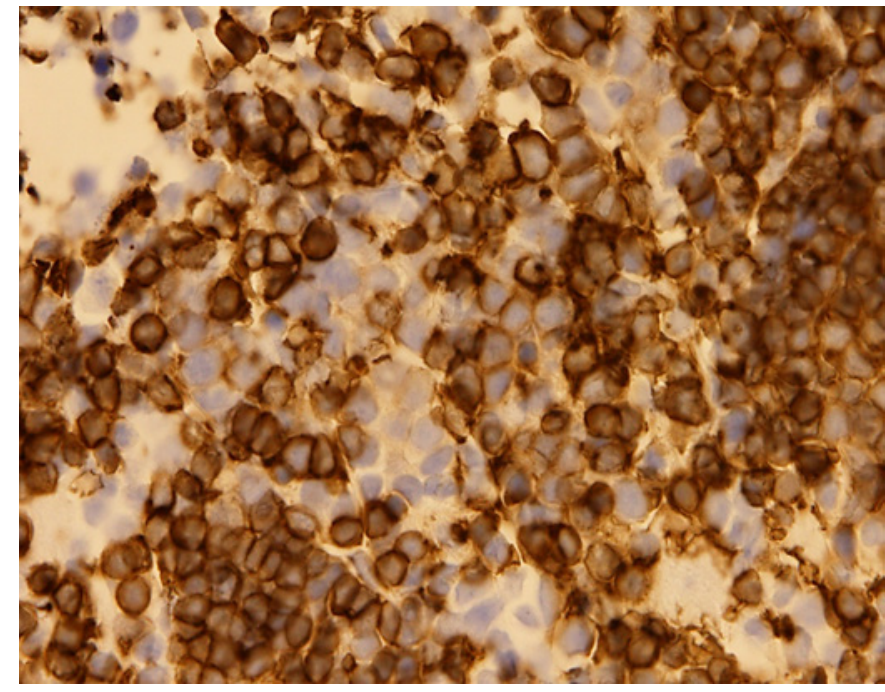

Fig. 3. Glycophorin staining of the mediastinal mass, showing an abundance of the erythroid lineage.

row aspirate showed no evidence of involvement by clonal B cell or $\mathrm{T}$ cell populations. The trephine biopsy showed trilineage hematopoiesis with marked erythroid and mild histiocytes hyperplasia. The histology of both the mediastinal mass and bone marrow was highly suggestive of EMH. However, immunohistochemistry was needed to exclude other possibilities, such as Langerhans cell histiocytosis, thymoma, and neurogenic tumor. Extensive immunostaining was performed. Myeloperoxidase and glycophorin confirmed the abundance of erythroid elements (Fig. 3). CD34 and CD117 immunostains showed no increase in blasts. Ki67 showed a variable proliferation rate mainly of the erythroid element. The histiocytic marker CD68 showed faint focal expression. CD20 showed scattered B cells. CD3 showed rare T cells. We detected no noted expression of S100, langerin, CD1a, CD23, or CD21. These results further confirmed the diagnosis of EMH and excluded the other possibilities.

\section{Differential Diagnoses}

1. Lymphoproliferative disorder, mainly non-Hodgkin's lymphoma, as the initial presentation was with pancytopenia, hepatosplenomegaly, and mediastinal mass

2. Thymoma

3. Langerhans cell histiocytosis

The histopathology and immune histochemistry excluded these possibilities.

\section{Treatment}

The patient was initially managed symptomatically by blood transfusion and antibiotics. After confirming the diagnosis, the patient was commenced on hydroxyurea ( $1 \mathrm{~g}$, orally) and folic acid.

Outcome and Follow-Up

The patient was discharged on hydroxyurea $(1 \mathrm{~g}$, once daily, orally) and folic acid. He was followed-up in the thalassemia center. His chest tightness and shortness of breath improved. The
WBC count recovered. The platelet count, however, remained lower than normal, ranging from 90 to $110 \times 10^{9} / \mathrm{L}$. He was started on a regular blood transfusion program. Repeated imaging demonstrated the persistence of the paramediastinal mass, yet the size of the mass decreased slightly. Right-sided pleural effusion persisted till 1 year after presentation, when it showed complete resolution by CT scan.

\section{Discussion}

The marrow located in the medullary cavity of the bone is the sole site of effective hematopoiesis in humans. Hematopoietically active (red) marrow regresses after birth until late adolescence, after which it is focused in the lower skull, vertebrae, shoulder and pelvic girdles, ribs, and sternum. EMH is a compensatory phenomenon of altered hematopoiesis occurring secondary to inadequate bone marrow function [1-3]. The causes of EMH can be pathological or physiological. Pathological causes include hemoglobinopathies, hemolytic anemias, leukemias, lymphomas, and myeloproliferative neoplasms [4-6]. $\mathrm{EMH}$ is commonly found in the liver and spleen. Less commonly involved organs include the pleura, lungs, gastrointestinal tract, breast, skin, central nervous system, kidneys, and adrenal glands [2, 7]. All these extramedullary sites are thought to be engaged in active hematopoiesis in fetal life, which normally stops at birth but can retain this function under long-standing circumstances, leading to ineffective RBC formation $[1,8]$.

In rare cases, EMH presents as a solitary mass, appearing as a tumor-simulating lesion in an atypical location, and is therefore frequently misdiagnosed. Tumors composed of hematopoietic tissue, sometimes with intense fibrosis, can develop in the bowel, breast, liver, lungs, mediastinum, pleura, and mesentery, skin, synovium, thymus, thyroid, thorax, prostate, spleen, urinary tract, and intracranial or intraspinal epidural space $[1,2,8]$. Symptoms and signs depend on the site of the tumor. Patients can sometimes be asymptomatic. If serosal surfaces are involved, patients might present with effusions, sometimes massive, in the thorax, abdomen, and pericardial space [2]. EMH tumors in the intracranial or intraspinal epidural space can lead to serious neurologic complications, including subdural hemorrhage, delirium, increased intracranial pressure, orbital apex syndrome, papilledema, cerebral tumor, coma, motor and sensory impairment, spinal cord compression, and limb paralysis $[2,8,9]$. EMH tumors can be diagnosed by tissue biopsy, CT scan, magnetic resonance imaging (MRI), or positron emission tomography after ${ }^{52} \mathrm{Fe}$ infusion $[1,2]$. The treat- 
ment of EMH tumors includes blood transfusion, radiotherapy, surgical decompression, hydroxyurea, possibly JAK2 inhibitors, or a combination of these modalities [1, $2,8]$. JAK2 inhibitors and TGF- $\beta$ ligand traps that ameliorate ineffective erythropoiesis processes are already being tested in clinical trials with promising results in patients with thalassemia and EMH [10].

Our patient presented with mediastinal EMH and pleural effusion. These masses rarely induce significant symptoms but might result in hemothorax and pleural effusion. The mechanism of developing pleural effusion is believed to be multifactorial. Mechanical lymphatic obstruction and liberation of inflammatory cytokines, which increase the capillary permeability during the friction between the pleu$\mathrm{ra}$ and the pseudotumor, seem to be contributing mechanisms [11]. Direct nontraumatic bleeding from the EMH mass into the pleural cavity is another mechanism [12]. In our patient, a lower respiratory tract infection might have contributed to the development of pleural effusion.

The clinical presentation of our patient mimicked other systemic disorders, having pancytopenia, hepatosplenomegaly, mediastinal mass, and pleural effusion. Therefore, it was necessary to have tissue diagnosis to exclude disorders like lymphoma.

Treatment of EMH is unnecessary in the absence of complications [2] and depends on the severity of symptoms, the nature of the underlying disease, the production rate of the pleural fluid, and the patient's clinical status [11]. The management of hemothorax caused by EMH should be individualized. It involves drainage of the fluid and conservative follow-up or pleurodesis for symptomatic recurrent pleural effusions. Local management of the pleural effusion should be combined with treatment of the underlying disease, and management of the EMH mass by either hydroxyurea, a hypertransfusion program, surgical resection or irradiation, or a combination of these modalities $[1,2,8]$. For our case, the pleural effusion was drained as he was symptomatic. Hydroxyurea was started. He was scheduled for a regular blood transfusion program. After $>1$ year of follow-up, our patient was clinically well. He did not develop any further episode of pleural effusion. His EMH mass is still present, yet slightly smaller.

In the literature, Peng et al. [13] reported a 34-year-old man with $\beta$-thalassemia intermedia who developed intrathoracic EMH with recurrent pleural effusion. The diagnosis was established by thoracoscopic biopsy. Pleurodesis with minocycline installation was used to control recurrent pleural effusion. Smith et al. [12] reported a case of a 49-year-old woman with thalassemia intermedia who developed massive hemothorax due to direct hemorrhage into the pleural cavity from a large intrathoracic paraspinal hematopoietic mass. Initially, thoracotomy was done to control bleeding. Then the extramedullary mass was managed by irradiation. This prevented further bleeding into the pleural cavity. Chu et al. [14] described a 44-yearold man with $\alpha$-thalassemia and massive left-sided pleural effusion, which was managed conservatively with initial drainage by chest tube and regular follow-up. Meo et al. [15] described 2 patients with thalassemia intermedia, a 48-year-old woman with intrathoracic and symptomatic pelvic EM masses and a 42-year-old male with bilateral intrathoracic masses causing respiratory insufficiency and pleural effusion. Both patients showed clinical improvement with hydroxyurea therapy and occasional blood transfusions. Chen et al. [5] described an 80-yearold lady with primary myelofibrosis and bilateral pleural effusion who responded fully to ruxolitinib after being refractory to hydroxyurea.

The variability of management options of intrathoracic $\mathrm{EMH}$, as demonstrated in the literature, confirms that management should be individualized according to the case. More research is required to discover useful strategies for preventing the development of EMH.

\section{Acknowledgements}

We thank Bassam Odeh (consultant hematopathologist, Dubai Hospital) for assistance with producing microscope images of the biopsy specimen.

\section{Statement of Ethics}

The authors have no ethical conflicts to disclose. Consent for publication was signed by the patient's father.

\section{Disclosure Statement}

The authors have no conflicts of interest to declare.

\section{Funding Sources}

No funding to be disclosed for this manuscript.

\section{Author Contributions}

Hasan AAL-Yaseen: clinician, data collection, writing and editing the manuscript; Mahmoud Marashi: clinician; Asma Sultan Al Olama: clinician; and Rania Seliem: histopathological diagnosis and slide image review. 


\section{References}

1 Musallam KM, Rivella S, Vichinsky E, Rachmilewitz EA. Non-transfusion-dependent thalassemias. Haematologica. 2013 Jun;98(6): 833-44.

2 Koch CA, Li CY, Mesa RA, Tefferi A. Nonhepatosplenic extramedullary hematopoiesis: associated diseases, pathology, clinical course, and treatment. Mayo Clin Proc. 2003 Oct; 78(10):1223-33.

$3 \mathrm{Kim} \mathrm{CH}$. Homeostatic and pathogenic extramedullary hematopoiesis. J Blood Med. 2010; $1: 13-9$.

4 Handin RI, Lux SE, Stossel TP. Blood: principles and practice of hematology. Philadelphia (PA): Lippincott Williams \& Wilkins; 2003. vol. 1, p. 395.

5 Chen K, Krveshi L, Ballarino G. Pleural extramedullary hematopoiesis successfully treated with ruxolitinib: A case report. Chest. 2017; 152(4 Supplement):A507.

6 Xiros N, Economopoulos T, Papageorgiou E, Mantzios G, Raptis S. Massive hemothorax due to intrathoracic extramedullary hematopoiesis in a patient with hereditary spherocytosis. Ann Hematol. 2001 Jan;80(1):38-40.
7 Karami H, Kosaryan M, Taghipour M, Sharifian R, Aliasgharian A, Motalebi M. Extramedullary hematopoiesis presenting as a right adrenal mass in a patient with $\beta$ thalassemia. Nephrourol Mon. 2014 Sep;6(5): e19465.

8 Haidar R, Mhaidli H, Taher AT. Paraspinal extramedullary hematopoiesis in patients with thalassemia intermedia. Eur Spine J. 2010 Jun;19(6):871-8.

9 Salehi SA, Koski T, Ondra SL. Spinal cord compression in beta-thalassemia: case report and review of the literature. Spinal Cord. 2004 Feb;42(2):117-23.

10 Makis A, Hatzimichael E, Papassotiriou I, Voskaridou E. 2017 Clinical trials update in new treatments of $\beta$-thalassemia. Am J Hematol. 2016 Nov;91(11):1135-45.

11 Papakala E, Porpodis K, Fouka E, Lagoudi K, Efthymiou C, Papakosta D. Pleural effusion as the main manifestation of intrathoracic extramedullary heamatopoietic masses in a patient with beta thalassemia minor. Pneumon. 2016; 29:156-60.
12 Smith PR, Manjoney DL, Teitcher JB, Choi $\mathrm{KN}$, Braverman AS. Massive hemothorax due to intrathoracic extramedullary hematopoiesis in a patient with thalassemia intermedia. Chest. 1988 Sep;94(3):658-60.

13 Peng MJ, Kuo HT, Chang MC. A case of intrathoracic extramedullary hematopoiesis with massive pleural effusion: successful pleurodesis with intrapleural minocycline. J Formos Med Assoc. 1994 May;93(5):445-7.

14 Chu KA, Lai RS, Lee CH, Lu JY, Chang HC, Chiang HT. Intrathoracic extramedullary haematopoiesis complicated by massive haemothorax in alpha-thalassaemia. Thorax. 1999 May;54(5):466-8.

15 Meo A, Cassinerio E, Castelli R, Bignamini D, Perego L, Cappellini MD. Effect of hydroxyurea on extramedullary haematopoiesis in thalassaemia intermedia: case reports and literature review. Int J Lab Hematol. 2008 Oct; 30(5):425-31. 